\title{
COMBINED KINETIC ANALYSIS OF THERMAL DEGRADATION OF POLYMERIC MATERIALS UNDER ANY THERMAL PATHWAY.
}

\author{
P.E Sánchez-Jiménez*, L.A. Pérez-Maqueda , A. Perejón and J.M. Criado. \\ Instituto de Ciencia de Materiales de Sevilla, C.S.I.C.-Universidad de Sevilla, C. Américo \\ Vespucio n²49, 41092 Sevilla, Spain
}

\begin{abstract}
Combined kinetic analysis has been applied for the first time to the thermal degradation of polymeric materials. The combined kinetic analysis allows the determination of the kinetic parameter from the simultaneous analysis of a set of experimental curves recorded under any thermal schedule. Besides, the method does not make any assumption about the kinetic model or activation energy and allows the analysis even when the process does not follow one of the ideal kinetic models already proposed in literature. In the present paper the kinetics of the thermal degradation of both polytetrafluoroethylene (PTFE) and polyethylene (PE) have been performed. It has been concluded, without previous assumptions on the kinetic model, that the thermal degradation of PTFE obeys a first order kinetic law, while the thermal degradation of PE follows a diffusion-controlled kinetic model.
\end{abstract}

Keywords: Kinetics, Polymer degradation, SCTA

\footnotetext{
* Corresponding author.

Current address: Department of Mechanical Engineering, University of Colorado at Boulder, Boulder CO 80309-0427 USA Tel +1 3037352651 Fax: +1 3034923498

Email addresses: Pedro.Sanchez@,colorado.edu (P.E. Sanchez), maqueda@cica.es ( L.A. Pérez-Maqueda)
} 


\section{Introduction}

Thermal stability studies of polymers are a matter of great interest because of the technical and commercial importance of these materials [1-5] with the kinetic analysis of thermal degradation playing an important role in such studies [6-22]. A reliable evaluation of the kinetic parameters permits a theoretical interpretation of the experimental data and provides a mathematical description needed to extrapolate the reaction behaviour to conditions different from the experimental ones [23-25].

Thermal analysis methods, mainly differential scanning calorimetry and thermogravimetry, are extensively used in thermal degradation studies of polymers. Experimental data for the kinetic analysis can be recorded under different experimental conditions. Thus, different temperature versus time programs, both isothermal and nonisothermal, have been proposed. In the isothermal experiment, samples should be rapidly heated up to the final temperature and maintained at this temperature while the thermal degradation is recorded as a function of the time. In this latter case, the low thermal conductivity of polymers makes it difficult to reach a steady state at the selected temperature before the reaction starts [26, 27]. Additionally, the length of the experiment is not known in advance and it will be determined by the selected temperature. Thus, this temperature should be properly chosen to avoid extremely long or short experiments. An alternative to isothermal experiment is the linear heating rate program, where temperature varies in a wide range of values while the thermal degradation is recorded as a function of the temperature. This linear heating rate program is the most widely used [28]. In any case, it is not uncommon to find in the literature a serious disagreement between the kinetic parameters calculated from data obtained under isothermal and non-isothermal conditions [26, 29, 30]. Sample 
Controlled Thermal Analysis (SCTA) constitutes an interesting non isothermal method which has been rarely applied to the kinetic analysis of thermal degradation of polymers [31]. In SCTA experiments, the evolution of the reaction rate with the time is predefined by the user and, most usually, it is maintained at a constant value along the entire process. In this case the technique is known as Constant Rate Thermal Analysis (CRTA). Thus, mass and heat transfer phenomena occurring during the decomposition process can be minimized by selecting a constant decomposition rate that is low enough, yielding results which are more representative of the forward reaction [31-33]. The pyrolysis of organic materials, such as polymers, is a chemically complex process, where several reactions may be occurring simultaneously [1, 34-36]. Because of this complexity, a large number of papers found in the literature assume first order or norder kinetic models to describe the thermal degradation of polymers $[8,23,30,34,37$ 45], what would entail a non realistic description of the real reaction leading to erroneous kinetic parameters. Combined kinetic analysis is a procedure recently proposed that allows for the simultaneous analysis of a set of experimental curves recorded under any thermal schedule [27, 46-48]. To overcome the difficulty of finding a kinetic model which is able to describe accurately the real process, the combined kinetic analysis procedure have been recently upgraded by the use of an empirical kinetic equation based on that proposed by Sestak-Berggren [49] that fits every kinetic function corresponding to the ideal models used in literature and their probable deviations from ideality [46]. Thus, the combined kinetic analysis can be used to obtain the kinetic triplet, i.e. activation energy, preexponential factor and kinetic mode, from a set of data obtained under different experimental conditions and without any previous assumption about the kinetic model or the activation energy of the process. 
The aim of this work is the application for the first time of the combined kinetic analysis to the study of two widely used commercial polymers, polytetrafluoroethylene (PTFE), and polyethylene (PE), proving that data obtained under different experimental conditions may all together be described by the same kinetic parameters. Moreover, the kinetic parameters obtained will be used to reconstruct the original curves in order to demonstrate their validity, something that is rarely done in most kinetic studies found in literature.

\section{Theoretical background.}

The reaction rate, $d \alpha / d t$, of a solid state reaction can be described by the following general equation [31]:

$$
\frac{d \alpha}{d t}=A \exp (-E / R T) f(\alpha)
$$

where $A$ is the Arrhenius preexponential factor, $R$ is the gas constant, $E$ the activation energy, $\alpha$ the reacted fraction, $T$ is the process temperature and $f(\alpha)$ accounts for the reaction rate dependence on $\alpha$. The kinetic model, $f(\alpha)$ is an algebraic expression which is usually associated with a physical model that describes the kinetics of the solid state reaction [50]. Table 1 show the functions corresponding to the most commonly used kinetic mechanisms found in literature.

Eq (1) is a general expression that describes the relationship among the reaction rate, reacted fraction and temperature independently of the thermal pathway used for 
recording the experimental data. In the case that the experimental data were recorded at a constant reaction rate $\beta=d T / d t, \mathrm{Eq}$ (1) can be written as follows [51]:

$$
\frac{d \alpha}{d T}=\frac{A}{\beta} \exp (-E / R T) f(\alpha)
$$

Under constant rate thermal analysis (CRTA) conditions, the reaction rate is maintained at a constant value $\mathrm{C}=\mathrm{d} \alpha / \mathrm{dt}$ selected by the user and $\mathrm{Eq}$ (1) becomes:

$$
C=A \exp (-E / R T) f(\alpha)
$$

\subsection{Isoconversional Analysis}

Isoconversional methods (model-free methods) are used for determining the activation energy as a function of the reacted fraction without any previous assumption on the kinetic model fitted by the reaction. The Friedman isoconversional method [52] is a widely used differential method that, unlike conventional integral model-free methods, provides accurate values of activation energies even if the activation were a function of the reacted fraction [53].

Eq (1) can be written in logarithmic form:

$$
\ln \left(\frac{d \alpha}{d t}\right)=\ln (A f(\alpha))-\frac{E}{R T}
$$

Moreover, at a constant value of $\alpha, f(\alpha)$ would be also constant and Eq (4) would be written in the form 


$$
\ln \left(\frac{d \alpha}{d t}\right)=\text { Const }-\frac{E}{R T}
$$

The activation energy at a constant $\alpha$ value can be determined from the slope of the plot of the left hand side of Eq (4) against the inverse of the temperature, at constant values of $\alpha$.

\subsection{Combined Kinetic Analysis}

The logarithmic form of the general kinetic equation (Eq (1)) can be written as follows:

$$
\ln \left(\frac{d \alpha / d t}{f(\alpha)}\right)=\ln A-\frac{E}{R T}
$$

If the proper $f(\alpha)$ function is selected, the plot of the left hand side of Eq (6) versus 1/T would yield a straight line whose slope leads to the activation energy, while the intercepts allows to determine the preexponential factor of Arrhenius once the activation energy is known. It is noteworthy to point out that the relationships among the triplet d $\alpha /$ dt- $\alpha-\mathrm{T}$ quoted by Eq (6) is independent of the thermal pathway used for reaching a particular value of the triplet, what leads to the conclusion that Eq (6) would allow the simultaneous analysis of any sets of experimental data obtained under different heating schedules [27].

There is an important limitation related with the way the proper $f(\alpha)$ function is selected. The $f(\alpha)$ kinetic equations proposed in literature have been developed assuming idealized physical conditions that would not be necessarily fulfilled by the real solid state reaction. Deviation from the ideal models described in Table 1 would be expected due to factors such as heterogeneous distribution in particle size, particle shapes, etc. To 
overcome this problem, a new procedure has been introduced in a recent work, where the following $f(\alpha)$ general expression was proposed [46]:

$$
f(\alpha)=c(1-\alpha)^{n} \alpha^{m}
$$

This equation is a modified form of the Sestak-Berggren empirical equation [49]. It has been shown that it can fit every function in Table 1 by merely adjusting the parameters c, $\mathrm{n}$ and $\mathrm{m}$ [46]. Therefore, Eq (7) works as an umbrella that covers the most common physical models and its possible deviations from ideal conditions. From Eq (6) and (7). we get

$$
\ln \left(\frac{d \alpha / d t}{(1-\alpha)^{n} \alpha^{m}}\right)=\ln c A-E / R T
$$

that should fit experimental data obtained under any heating schedule. The Pearson linear correlation coefficient between the left hand side of the equation and the inverse of the temperature is set as an objective function for optimization. By means of the

maximize function of the software Mathcad (PTC), parameters $\mathrm{n}$ and $\mathrm{m}$ that yield the best linear correlation are obtained and the corresponding values of $\mathrm{E}$ and $\mathrm{A}$ can be calculated.

\section{Experimental}

The following commercial polymers were studied: polytetrafluoroethylene (Aldrich, product number 182478) and polyethylene (Aldrich, product number 332119). 
Thermogravimetry measurements were carried out with homemade TGA instrument that uses a CI Electronics Ltd electrobalance connected to a gas flow system to work in inert atmosphere $\left(70 \mathrm{cc} \mathrm{min}^{-1} \mathrm{~N}_{2}\right)$. Small samples $(9 \mathrm{mg})$ were used in order to minimize heat and mass transfer phenomena. They were placed on a $1 \mathrm{~cm}$ diameter platinum pan inside a low thermal inertia homemade furnace. The instrument allows working either under conventional linear heating conditions or under sample controlled conditions. A description of the experimental set-up can be found in references [54-56]. A set of thermal degradation curves, obtained under both linear heating rate and constant rate controlled conditions, were carried out for each polymer. Experimental integral curves were differentiated by means of the Origin software (OriginLab) to obtain the differential curves required for the kinetic analysis.

\section{Results and discussion}

\subsection{Polytetrafluoroethylene}

Fig 1 shows the experimental curves recorded for the thermal degradation of PTFE under linear heating rate (1a) and sample controlled (1b) conditions. Linear heating rate

experiments were carried out at 1,2 and $5^{\circ} \mathrm{C} \min ^{-1}$, while sample controlled were conducted at reaction rates of $510^{-4}$ and $8.310^{-4} \mathrm{~min}^{-1}$.

In Fig 2, the $510^{-4} \mathrm{~min}^{-1}$ CRTA curve is presented as an example of the kind of experimental curves obtained under constant rate experimental conditions. Both the temperature and the reacted fraction are plotted as a function of time as directly recorded by the instrument. It can be observed that the temperature rises until reaching 
the constant reaction rate previously selected with our experimental arrangement forcing $\alpha$ to fits a straight line as a function of the time.

Fig 3 shows several of the isoconversional Friedman plots obtained from the simultaneous analysis, according with Eq (4), of the $\alpha$-T curves shown in Fig 1 that were obtained under constant heating rate and CRTA heating schedules, respectively. The values of the activation energy calculated from the slope of the Friedman plots for different $\alpha$ values are included in Table 2 together with their corresponding linear correlation coefficients. These results demonstrate that a constant activation energy $\mathrm{E}=$ $286 \mathrm{~kJ} \mathrm{~mol}^{-1}$ has been obtained along the entire $\alpha$ range.

The combined kinetic analysis of these curves was performed by means of eq (8). Fig 4 shows the simultaneous plot of the values calculated for the left hand side of eq (8) from the whole set of experimental data in Fig 1 versus $1 / \mathrm{T}$. It is clearly shown that all experimental data are fitted by a single straight line with $n=0.901$ and $m=-0.081$, giving a correlation coefficient of 0.999 . The slope of the plot leads to an activation energy value of $283 \pm 2 \mathrm{~kJ} / \mathrm{mol}$ and the intercept to an Arrhenius pre-exponential factor of $(4.1 \pm 1.1) 10^{16}$. It must be pointed out that the activation energy obtained closely agrees with the one calculated from the Friedman isoconversional analysis without any previous assumption of the kinetic model.

Fig 5 shows the comparison of the $f(\alpha)$ function resulting from the combined analysis with some of the conversion functions often used in the literature, which are listed in Table 1. It is clear from this figure that the conversion function associated with the thermal degradation of PTFE closely follows a F1 kinetic model.

The simulation of curves obtained with the calculated kinetic parameters is a useful method for checking the results obtained by this kinetic analysis. Thus, a set of curves have been simulated assuming identical heating conditions as those used in the 
experiments and the kinetic parameters obtained from the combined kinetic analysis. The simulations have been performed from Eq (1) and the equations that define the heating conditions, i.e. linear heating or constant rate. As it is shown in Fig 1, both the reconstructed (simulated) curves and the experimental ones match almost exactly, proving the validity of the kinetic parameters obtained from the analysis. Additionally, curves obtained under different experimental conditions are expected to be affected by different heat and mass transfer phenomena. The fact that all of the curves are reconstructed with the same kinetic parameters seems to indicate that these mass and heat transfer phenomena have been succesfully minimized.

\subsection{Polyethylene}

Fig 6 shows the experimental curves recorded for the thermal degradation of polyethylene under linear heating rate (6a) and sample controlled (6b) conditions. Linear heating rate experiments were carried out at $0.5,1,2$ and $10{ }^{\circ} \mathrm{C} \mathrm{min}^{-1}$, while sample controlled experiments were conducted at constant decomposition rates of 1.6 $10^{-3}$ and $8.310^{-4} \mathrm{~min}^{-1}$, respectively.

Fig 7 shows some of the isoconversional Friedman plots obtained at different values of $\alpha$ from the simultaneous analysis of the $\alpha$-T plots obtained under linear heating and CRTA temperature control, respectively, that are shown in Fig 6. The values of E obtained for different $\alpha$ values are included in Table 3 together with their corresponding correlations coefficients. These results allow to conclude that a constant activation energy $\mathrm{E}=253 \mathrm{~kJ} \mathrm{~mol}^{-1}$ is obtained all over the thermal decomposition range of polyethylene.

Fig 8 shows the result of the simultaneous combined kinetic analysis, by means of Eq (8), of the whole set of curves obtained under different heating schedules for the thermal 
decomposition of polyethylene that are reported in Fig 6. All experimental data are fitted by a single straight line when $n$ is 0.641 and $m$ is -0.646 , with a correlation factor of 0.997 . The slope of the plot leads to an activation energy value of $246 \pm 1 \mathrm{~kJ} \mathrm{~mol}^{-1}$ and the intercept to an Arrhenius preexponential factor of $(3.2 \pm 0.4) 10^{16} \mathrm{~min}^{-1}$. Fig 9 presents the comparison between the function $f(\alpha)$ obtained from the combined analysis and some of the more frequently used conversion functions used in the literature, showing that the conversion function associated to the thermal degradation of polyethylene approximately fits the master plot of diffusion controlled kinetic models. As in the case of the PTFE, the kinetic parameters resulting of the combined kinetic analysis have been used to simulate all the degradation curves shown in Fig 6 and, as it can be observed, both reconstructed and experimental curves match nicely, proving the validity of these kinetic parameters.

\section{Conclusions}

In this work, the method of combined kinetic analysis has been applied to study the thermal degradation of two commercial polymers, polytetrafluoroethylene and polyethylene. Experimental curves obtained under linear heating and sample controlled conditions have been analyzed simultaneously, obtaining kinetic parameters which can be used to successfully reconstruct all the curves. The close match between the reconstructed curves and the original ones proves the validity of the kinetic analysis method here proposed.

Additionally, it has been demonstrated that the "n order" kinetic law cannot be used as a universal model for describing the thermal degradation of polymers as frequently assumed in literature. The assumptions of unrealistic kinetic models would lead to 
wrong values of the kinetic parameters, making impossible the extrapolation to conditions different to those used for obtaining the experimental data.

\section{ACKNOWLEDGEMENT}

Financial support from projects TEP-03002 from Junta de Andalucía and MAT 200806619/MAT from the Spanish Ministerio de Ciencia e Innovación is acknowledged.

\section{References}

1. Pielichowski J and Njuguna J, editors. Thermal Degradation of Polimeric Materials. Shawbury: Rapra Technology Ltd, 2005.

2. Capone C, Di Landro L, Inzoli F, Penco M, and Sartore L. Thermal and mechanical degradation during polymer extrusion processing. Polym. Eng. Sci. 2007;47(11):1813-1819.

3. Dobkowski Z. Thermal analysis techniques for characterization of polymer materials. Polym. Degrad. Stabil. 2006;91(3):488-493.

4. Golebiewski J and Galeski A. Thermal stability of nanoclay polypropylene composites by simultaneous DSC and TGA Composites Sci. Tech. $2007 ; 67: 3442-3447$.

5. Hamdani S, Longuet C, Perrin D, Lopez-Cuesta JM, and Ganachaud F. Flame retardancy of silicone-based materials. Polym. Degrad. Stabil. 2009;94(4):465495. 
6. Levine SE and Broadbelt LJ. Detailed mechanistic modeling of high-density polyethylene pyrolysis: Low molecular weight product evolution. Polym. Degrad. Stabil. 2009;94(5):810-822.

7. Jimenez M, Duquesne S, and Bourbigot S. Kinetic analysis of the thermal degradation of an epoxy-based intumescent coating. Polym. Degrad. Stabil. 2009;94(3):404-409.

8. Gao ZM, Kaneko T, Hou DY, and Nakada M. Kinetics of thermal degradation of poly(methyl methacrylate) studied with the assistance of the fractional conversion at the maximum reaction rate. Polym. Degrad. Stabil. 2004;84(3):399-403.

9. Vyazovkin S, Dranca I, Fan XW, and Advincula R. Kinetics of degradation and relaxation of polystyrene clay nanocomposite. Abstracts of Papers of the American Chemical Society 2004;227:U559-U559.

10. Vyazovkin S and Sbirrazzuoli N. Isoconversional kinetic analysis of thermally stimulated processes in polymers. Macrom. Rapid Com. 2006;27(18):15151532.

11. Peltzer M, Wagner JR, and Jimenez A. Thermal characterization of UHMWPE stabilized with natural antioxidants. J. Therm. Anal. Cal. 2007;87(2):493-497.

12. Reverte C, Dirion JL, and Cabassud M. Kinetic model identification and parameters estimation from TGA experiments. J. Anal. Appl. Pyrol. 2007;79(12):297-305.

13. Erceg M, Kovacic T, and Klaric I. Poly(3-hydroxybutyrate) nanocomposites: Isothermal degradation and kinetic analysis. Thermochim. Acta 2009;485(12):26-32. 
14. Abate L, Bottino FA, Calanna S, and Pollicino A. Kinetic study of the thermal and oxidative degradations of poly(arylenether)s containing quinoline units. Polymer 1999;40(10):2719-2726.

15. Camino G, Lomakin SM, and Lazzari M. Polydimethylsiloxane thermal degradation - Part 1. Kinetic aspects. Polymer 2001;42(6):2395-2402.

16. Gao ZM, Wang HX, and Nakada M. Iterative method to improve calculation of the pre-exponential factor for dynamic thermogravimetric analysis measurements. Polymer 2006;47(5):1590-1596.

17. Han MG and Kim S. Controlled degradation of poly(ethyl cyanoacrylate-comethyl methacrylate) (PECA-co-PMMA) copolymers. Polymer 2009;50(5):1270-1280.

18. Kelsey DR, Kiibler KS, and Tutunjian PN. Thermal stability of poly(trimethylene terephthalate). Polymer 2005;46(21):8937-8946.

19. Perez-Maqueda LA, Sanchez-Jimenez PE, and Criado JM. Evaluation of the integral methods for the kinetic study of thermally stimulated processes in polymer science. Polymer 2005;46(9):2950-2954.

20. Budrugeac P. Some methodological problems concerning the kinetic analysis of non-isothermal data for thermal and thermo-oxidative degradation of polymers and polymeric materials. Polym. Degrad. Stabil. 2005;89(2):265-273.

21. Budrugeac P and Segal E. Application of isoconversional and multivariate nonlinear regression methods for evaluation of the degradation mechanism and kinetic parameters of an epoxy resin. Polym. Degrad. Stabil. 2008;93(6):10731080.

22. Budrugeac P, Segal E, Perez-Maqueda LA, and Criado JM. The use of the IKP method for evaluating the kinetic parameters and the conversion function of the 
thermal dehydrochlorination of PVC from non-isothermal data. Polym. Degrad. Stabil. 2004;84(2):311-320.

23. Paik P and Kar KK. Thermal degradation kinetics and estimation of lifetime of polyethylene particles: Effects of particle size. Materials Chemistry and Physics 2009;113(2-3):953-961.

24. Simon P, Cibulkova Z, and Thomas P. Accelerated thermooxidative ageing tests and their extrapolation to lower temperatures. J. Therm. Anal. Cal. $2005 ; 80(2): 381-385$.

25. Vyazovkin S. Model-free kinetics - Staying free of multiplying entities without necessity. J. Therm. Anal. Cal. 2006;83(1):45-51.

26. Maciejewski M. Computational aspects of kinetic analysis. Part B: The ICTAC Kinetics Project - the decomposition kinetics of calcium carbonate revisited, or some tips on survival in the kinetic minefield. Thermochim. Acta 2000;355(12):145-154.

27. Perez-Maqueda LA, Criado JM, Gotor FJ, and Malek J. Advantages of combined kinetic analysis of experimental data obtained under any heating profile. J. Phys. Chem. A 2002;106(12):2862-2868.

28. Khawam A and Flanagan DR. Basics and applications of solid-state kinetics: A pharmaceutical perspective. J. Pharm. Sci. 2006;95(3):472-498.

29. Brown ME, Maciejewski M, Vyazovkin S, Nomen R, Sempere J, Burnham A, Opfermann J, Strey R, Anderson HL, Kemmler A, Keuleers R, Janssens J, Desseyn HO, Li CR, Tang TB, Roduit B, Malek J, and Mitsuhashi T. Computational aspects of kinetic analysis Part A: The ICTAC kinetics projectdata, methods and results. Thermochim. Acta 2000;355(1-2):125-143. 
30. Ceamanos J, Mastral JF, Millera A, and Aldea ME. Kinetics of pyrolysis of high density polyethylene. Comparison of isothermal and dynamic experiments. J. Anal. Appl. Pyrol. 2002;65(2):93-110.

31. Criado JM and PerezMaqueda LA In: Sorensen OT and Rouquerol J, editors. Sample Controlled Thermal Analysis: Origins, Goals, Multiple Forms, Applications and Future, vol. 3: SCTA and Kinetics. Dordecht: Kluwer, 2003. p. $55-88$

32. Criado JM, Gotor FJ, Ortega A, and Real C. The New Method of Constant Rate Thermal-Analysis (Crta) - Application to Discrimination of the Kinetic-Model of Solid-State Reactions and the Synthesis of Materials. Thermochim. Acta 1992;199:235-238.

33. Sorensen OT and Rouquerol.J, editors. Sample Controlled Thermal Analysis. Budapest: Kluwer, 2003.

34. Bockhorn H, Hornung A, Hornung U, and Schwaller D. Kinetic study on the thermal degradation of polypropylene and polyethylene. J. Anal. Appl. Pyrol. 1999;48(2):93-109.

35. Conesa JA, Marcilla A, Caballero JA, and Font R. Comments on the validity and utility of the different methods for kinetic analysis of thermogravimetric data. J. Anal. Appl. Pyrol. 2001;58:617-633.

36. Marongiu A, Faravelli T, and Ranzi E. Detailed kinetic modeling of the thermal degradation of vinyl polymers. J. Anal. Appl. Pyrol. 2007;78(2):343-362.

37. Conesa JA and Font R. Polytetrafluoroethylene decomposition in air and nitrogen. Polym. Eng. Sci. 2001;41(12):2137-2147.

38. Gao ZM, Amasaki I, and Nakada M. A thermogravimetric study on thermal degradation of polyethylene. J. Anal. Appl. Pyrol. 2003;67(1):1-9. 
39. Inoue E, Tsuchiya M, Ishimaru K, and Kojima T. Thermogravimetric studies on poly(methyl methacrylate), poly(tetrahydrofuran) and their blends. J. Therm. Anal. Cal. 2002;70(3):747-753.

40. Liu NA and Fan WC. Critical consideration on the Freeman and Carroll method for evaluating global mass loss kinetics of polymer thermal degradation (vol 388, pg 85, 1999). Thermochim. Acta 2000;351(1-2):183-183.

41. Lu L, Yu HY, Wang SF, and Zhang Y. Thermal Degradation Behavior of Styrene-Butadiene-Styrene Tri-Block Copolymer/Multiwalled Carbon Nanotubes Composites. Journal of Applied Polymer Science 2009;112(1):524531.

42. Nishida H, Yamashita M, Hattori N, Endo T, and Tokiwa Y. Thermal decomposition of poly(1,4-dioxan-2-one). Polym. Degrad. Stabil. 2000;70(3):485-496.

43. Westerhout RWJ, Waanders J, Kuipers JAM, and vanSwaaij WPM. Kinetics of the low-temperature pyrolysis of polyethene, polypropene, and polystyrene modeling, experimental determination, and comparison with literature models and data. Industrial \& Engineering Chemistry Research 1997;36(6):1955-1964.

44. Al-Mulla A and Shaban HI. Thermal Degradation of Poly(Trimethylene Terephthalate) and Acrylonitrile Butadiene Styrene Blends: Kinetic Analysis of Thermogravimetric Data. Int. J. Polym. Mat. 2008;57(3):275-287.

45. Garcia N, Hoyos M, Guzman J, and Tiemblo P. Comparing the effect of nanofillers as thermal stabilizers in low density polyethylene. Polym. Degrad. Stabil. 2009;94(1):39-48.

46. Perez-Maqueda LA, Criado JM, and Sanchez-Jimenez PE. Combined kinetic analysis of solid-state reactions: A powerful tool for the simultaneous 
determination of kinetic parameters and the kinetic model without previous assumptions on the reaction mechanism. J. Phys. Chem. A 2006;110(45):1245612462.

47. Criado JM, Perez-Maqueda LA, Gotor FJ, Malek J, and Koga N. A unified theory for the kinetic analysis of solid state reactions under any thermal pathway. J. Therm. Anal. Cal. 2003;72(3):901-906.

48. Perez-Maqueda LA, Criado JM, and Malek J. Combined kinetic analysis for crystallization kinetics of non-crystalline solids. J. Non-Cryst. Solids 2003;320(1-3):84-91.

49. Sestak J and Berggren G. study of the kinetics of the mechanism of solid-state reactions at increased temperature. Thermochim. Acta 1971;3:1-12.

50. Khawam A and Flanagan DR. Solid-state kinetic models: Basics and mathematical fundamentals. J. Phys. Chem. B 2006;110(35):17315-17328.

51. Criado JM and Perez-Maqueda LA. Sample controlled thermal analysis and kinetics. J. Therm. Anal. Cal. 2005;80(1):27-33.

52. Friedman HL. Kinetics of Thermal Degradation of Char-Forming Plastics from Thermogravimetry. Application to Phenolic Plastic J. Polym. Sci. C-Polym. Symp. 1964(6PC):183-\&.

53. Criado JM, Sanchez-Jimenez PE, and Perez-Maqueda LA. Critical study of the isoconversional methods of kinetic analysis. J. Therm. Anal. Cal. 2008;92(1):199-203.

54. Alcala MD, Criado JM, Gotor FJ, Ortega A, Perez Maqueda LA, and Real C. Development of a new thermogravimetric system for performing constant rate thermal analysis (CRTA) under controlled atmosphere at pressures ranging from vacuum to 1 bar. Thermochim. Acta 1994;240(1-2):167-173. 
55. Criado JM, Perez-Maqueda LA, Dianez MJ, and Sanchez-Jimenez PE.

Development of a universal constant rate thermal analysis system for being used with any thermoanalytical instrument. J. Therm. Anal. Cal. 2007;87(1):297-300.

56. Dianez MJ, Perez-Maqueda LA, and Criado JM. Direct use of the mass output of a thermobalance for controlling the reaction rate of solid-state reactions.

Review Sci. Inst. 2004;75(8):2620-2624. 


\section{Figures Caption}

Fig. 1. Experimental curves (solid lines) obtained for the thermal decomposition of polytetrafluoroethylene under the following experimental conditions: (a) linear heating rate of 1,2 and $5{ }^{\circ} \mathrm{C} \min ^{-1}$ and (b) sample controlled degradation rate of $510^{-4}$ and 8.3 $10^{-4} \mathrm{~min}^{-1}$. Reconstructed curves using the kinetic parameters obtained from the kinetic combined analysis are plotted as dots.

Fig. 2. Experimental curve corresponding to the thermal decomposition of PTFE under constant reaction rate conditions of $510^{-4} \mathrm{~min}^{-1}$.

Fig. 3. Friedman plots resulting of the isoconversional analysis for some selected $\alpha$ values of the experimental curves presented in Fig. 1.

Fig. 4. Combined kinetic analysis of experimental curves included in Fig. 1 by means of Eq. (8) for the $n$ and $m$ parameters resulting of the optimization procedure, i.e. $n=0.901$ and $m=-0.081$.

Fig. 5. Comparison of the $\mathrm{f}(\alpha)$ functions (solid lines) normalized at $\alpha=0.5$ corresponding to some of the ideal kinetic models included in Table 1 with the reduced Sestak-Berggren equation (dots) and the resulting values of $n$ and $m$ parameters, i.e. $n=$ 0.901 and $m=-0.081$, for the thermal degradation of polytetrafluoroethylene.

Fig. 6. Experimental curves (solid lines) obtained for the thermal decomposition of polyethylene under the following experimental conditions: (a) linear heating rate of 0.5 , 
1,2 and $10^{\circ} \mathrm{C} \min ^{-1}$ and (b) sample controlled degradation rate of $8.310^{-4}$ and $1.610^{-3}$ $\min ^{-1}$. Reconstructed curves using the kinetic parameters obtained from the kinetic combined analysis are plotted as dots.

Fig. 7. Friedman plots resulting of the isoconversional analysis for some selected $\alpha$ values of the experimental curves presented in Fig. 6.

Fig. 8. Combined kinetic analysis of curves included in Fig. 6 by means of Eq. (8) for the $\mathrm{n}$ and $\mathrm{m}$ parameters resulting of the optimization procedure, i.e. $n=0.641$ and $m=-$ 0.646 .

Fig. 9. Comparison of the $f(\alpha)$ functions (solid lines) normalized at $\alpha=0.5$ corresponding to some of the ideal kinetic models included in Table 1 with the reduced Sestak-Berggren equation (dotted line) and the resulting values of $n$ and $m$ parameters, i.e. $n=0.641$ and $m=-0.646$, for the thermal degradation of polyethylene. 
TABLE 1. $f(\alpha)$ kinetic functions for the most widely used kinetic models

\begin{tabular}{lll}
\hline Mechanism & Symbol & $\mathbf{f}(\alpha)$ \\
\hline
\end{tabular}

Phase boundary controlled reaction (contracting area)

$\mathrm{R} 2$

$$
(1-\alpha)^{1 / 2}
$$

Phase boundary controlled reaction (contracting volume)

R3 $\quad(1-\alpha)^{2 / 3}$

Random nucleation followed by an instantaneous growth of nuclei. (Avrami-Erofeev eqn. $n=1$ )

F1

$$
(1-\alpha)
$$

Random nucleation and growth of nuclei through different nucleation and nucleus growth models. (Avrami-Erofeev eqn.)

Two-dimensional diffusion

D2

$$
1 /[-\ln (1-\alpha)]
$$

Three-dimensional diffusion (Jander equation)

D3

$$
\frac{3(1-\alpha)^{2 / 3}}{2\left[1-(1-\alpha)^{1 / 3}\right]}
$$

Three-dimensional diffusion (Ginstling-Brounshtein equation)

D4 $\frac{3}{2\left[(1-\alpha)^{-1 / 3}-1\right]}$


TABLE 2. Activation energy values for different values of conversion and their correlation coefficients, obtained by the Friedman isoconversional analysis of the curves showed in Fig. 1.

\begin{tabular}{ccc}
\hline $\boldsymbol{\alpha}$ & $\mathbf{r}$ & $\mathbf{E a}\left(\mathbf{k J} \mathbf{~ m o l}^{-1}\right)$ \\
\hline 0.1 & 0.991 & $298 \pm 16$ \\
0.2 & 0.996 & $288 \pm 13$ \\
0.3 & 0.995 & $287 \pm 14$ \\
0.4 & 0.995 & $287 \pm 14$ \\
0.5 & 0.997 & $282 \pm 11$ \\
0.6 & 0.997 & $282 \pm 11$ \\
0.7 & 0.996 & $280 \pm 13$ \\
0.8 & 0.996 & $283 \pm 12$ \\
0.9 & 0.997 & $286 \pm 13$ \\
\hline
\end{tabular}


TABLE 3. Activation energy values for different values of conversion and their correlation coefficients, obtained by the Friedman isoconversional analysis of the curves showed in Fig. 6.

\begin{tabular}{ccc}
\hline $\boldsymbol{\alpha}$ & $\mathbf{r}$ & $\mathbf{E a}\left(\mathbf{k J ~ \mathbf { ~ m o l } ^ { - 1 } )}\right.$ \\
\hline 0.1 & 0.997 & $241 \pm 10$ \\
0.2 & 0.996 & $246 \pm 11$ \\
0.3 & 0.997 & $252 \pm 10$ \\
0.4 & 0.997 & $255 \pm 10$ \\
0.5 & 0.997 & $254 \pm 9$ \\
0.6 & 0.998 & $259 \pm 8$ \\
0.7 & 0.998 & $257 \pm 7$ \\
0.8 & 0.998 & $254 \pm 8$ \\
0.9 & 0.998 & $257 \pm 8$ \\
\hline
\end{tabular}



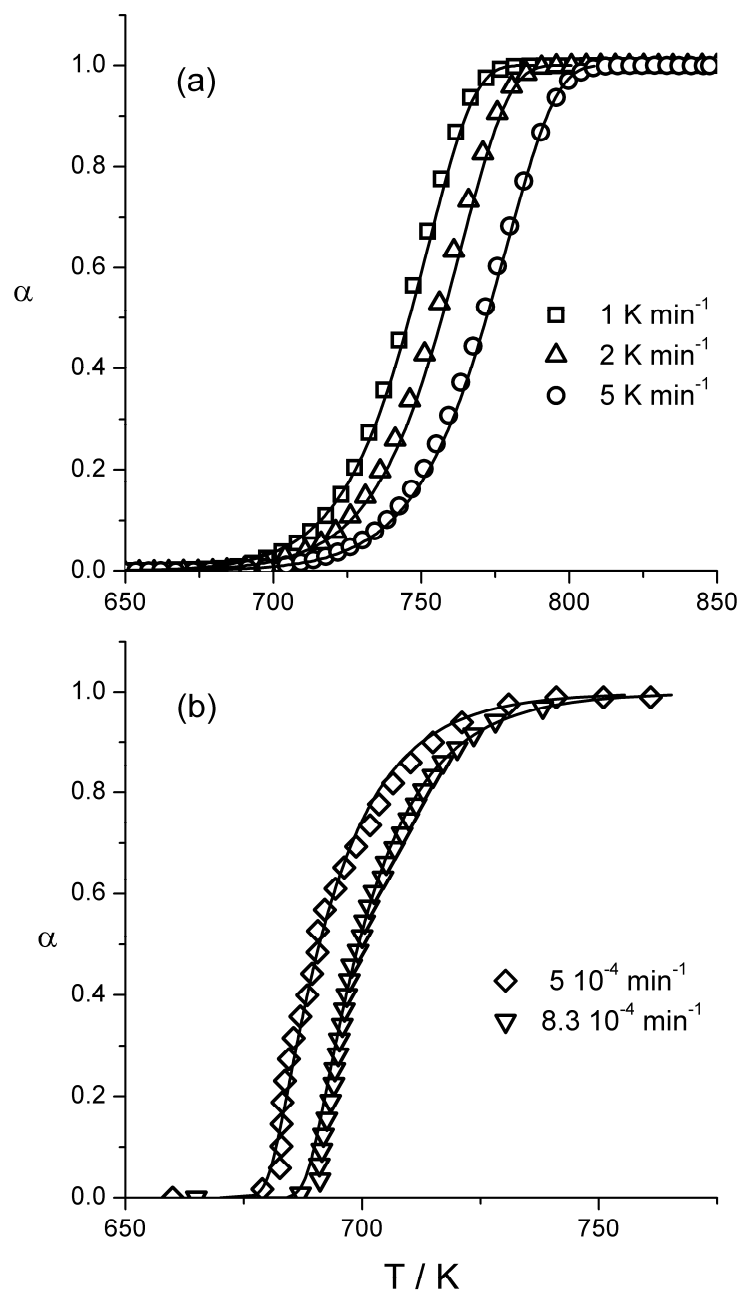

Fig. 1. Experimental curves (solid lines) obtained for the thermal decomposition of polytetrafluoroethylene under the following experimental conditions: (a) linear heating rate of 1,2 and $5{ }^{\circ} \mathrm{C} \min ^{-1}$ and (b) sample controlled degradation rate of $510^{-4}$ and 8.3 $10^{-4} \mathrm{~min}^{-1}$. Reconstructed curves using the kinetic parameters obtained from the kinetic combined analysis are plotted as dots. 


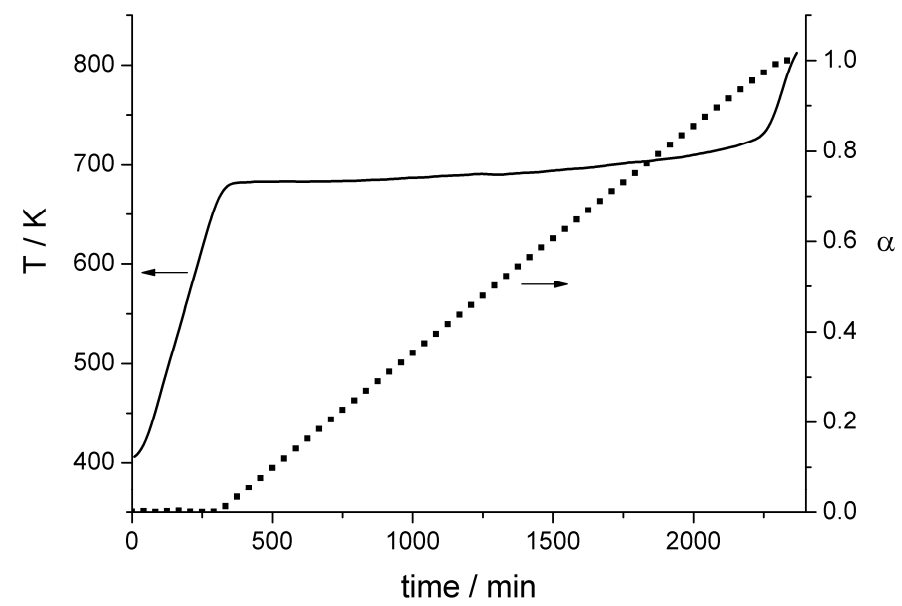

Fig. 2. Experimental curve corresponding to the thermal decomposition of PTFE under constant reaction rate conditions of $510^{-4} \mathrm{~min}^{-1}$.

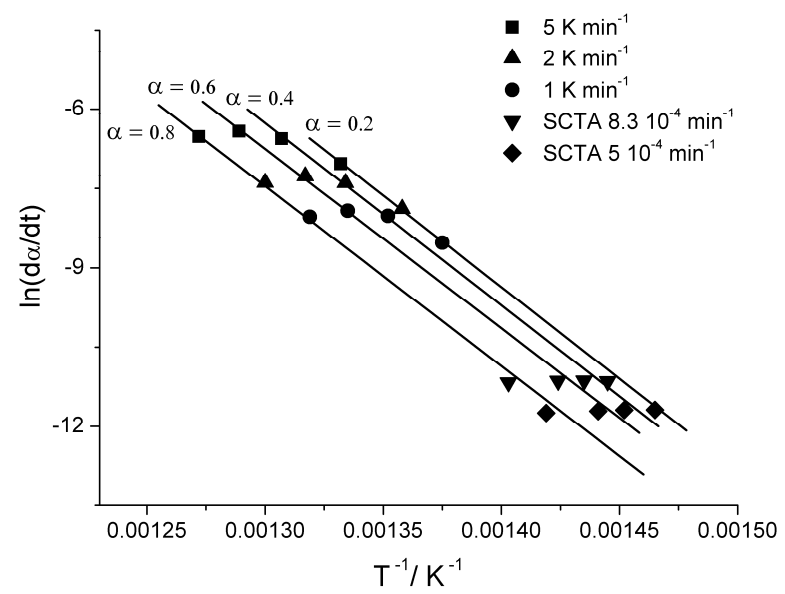

Fig. 3. Friedman plots resulting of the isoconversional analysis for some selected $\alpha$ values of the experimental curves presented in Fig. 1. 


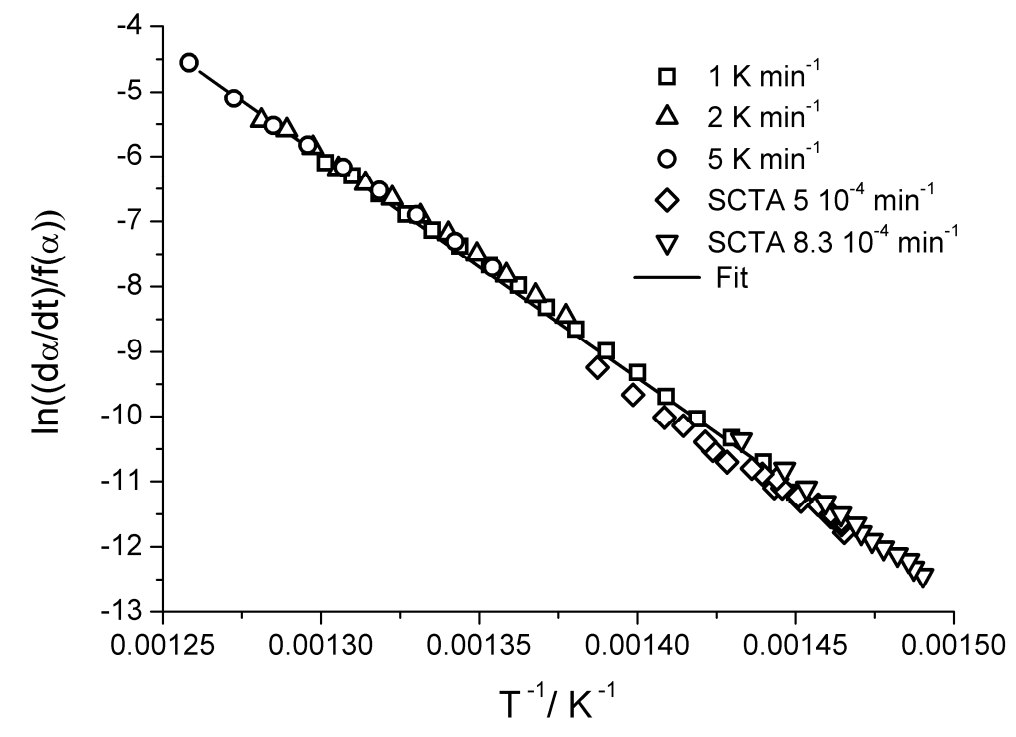

Fig. 4. Combined kinetic analysis of experimental curves included in Fig. 1 by means of Eq. (8) for the $\mathrm{n}$ and $\mathrm{m}$ parameters resulting of the optimization procedure, i.e. $n=0.901$ and $m=-0.081$.

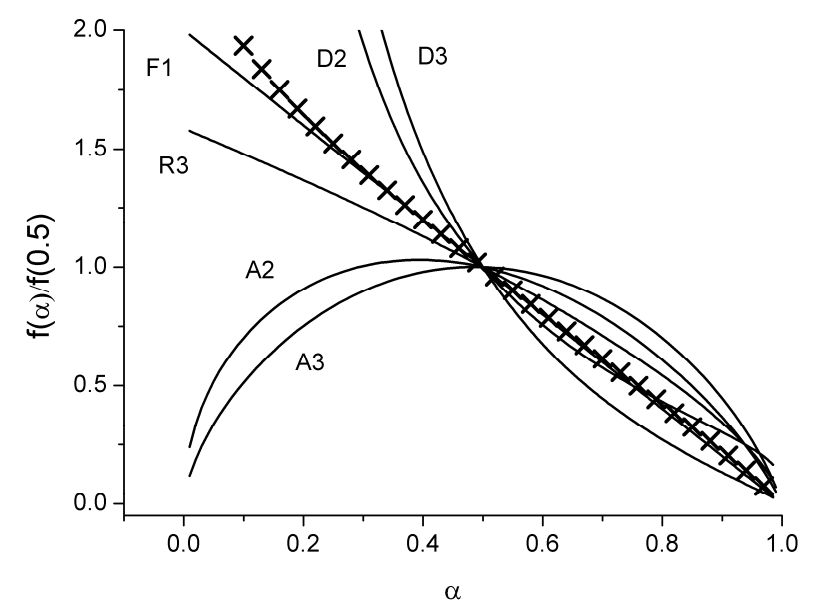

Fig. 5. Comparison of the $\mathrm{f}(\alpha)$ functions (solid lines) normalized at $\alpha=0.5$ corresponding to some of the ideal kinetic models included in Table 1 with the reduced Sestak-Berggren equation (dots) and the resulting values of $n$ and $m$ parameters, i.e. $n=$ 0.901 and $m=-0.081$, for the thermal degradation of polytetrafluoroethylene. 

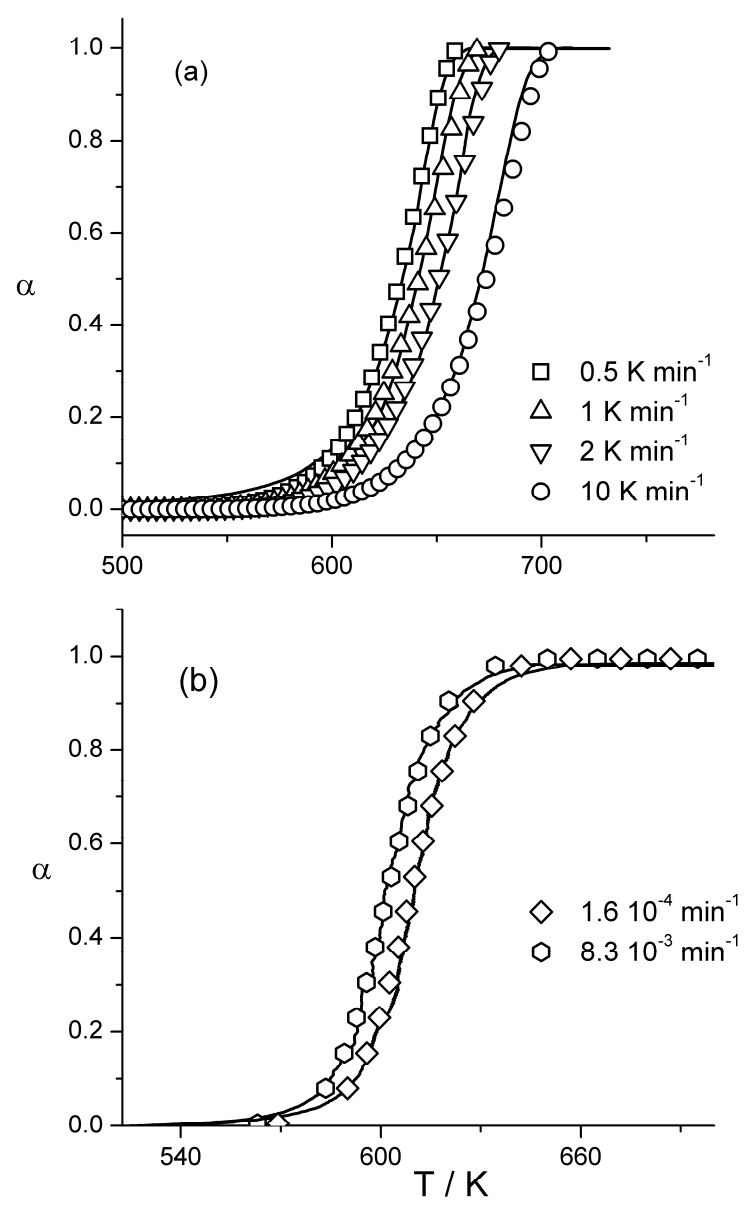

Fig. 6. Experimental curves (solid lines) obtained for the thermal decomposition of polyethylene under the following experimental conditions: (a) linear heating rate of 0.5 , 1,2 and $10^{\circ} \mathrm{C} \mathrm{min}^{-1}$ and (b) sample controlled degradation rate of $8.310^{-4}$ and $1.610^{-3}$ $\min ^{-1}$. Reconstructed curves using the kinetic parameters obtained from the kinetic combined analysis are plotted as dots. 


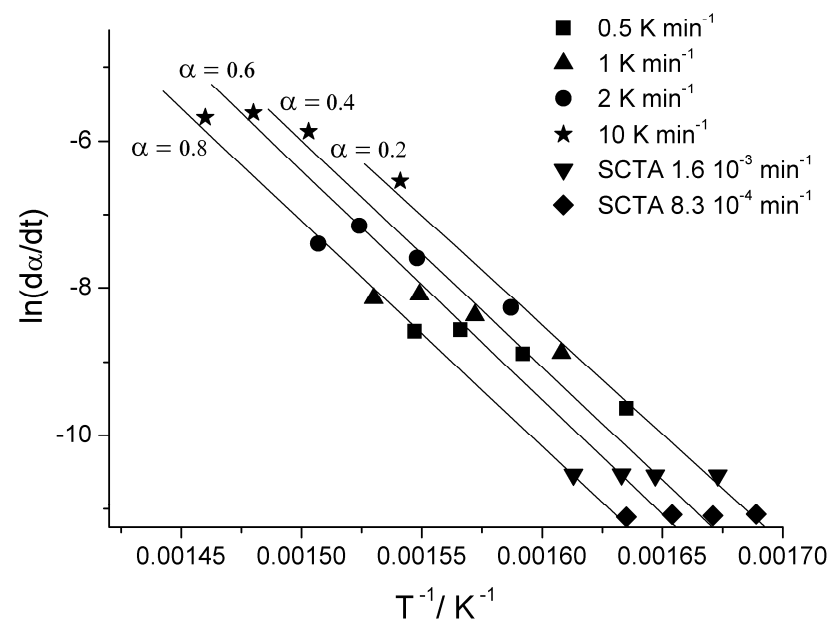

Fig. 7. Friedman plots resulting of the isoconversional analysis for some selected $\alpha$ values of the experimental curves presented in Fig. 6.

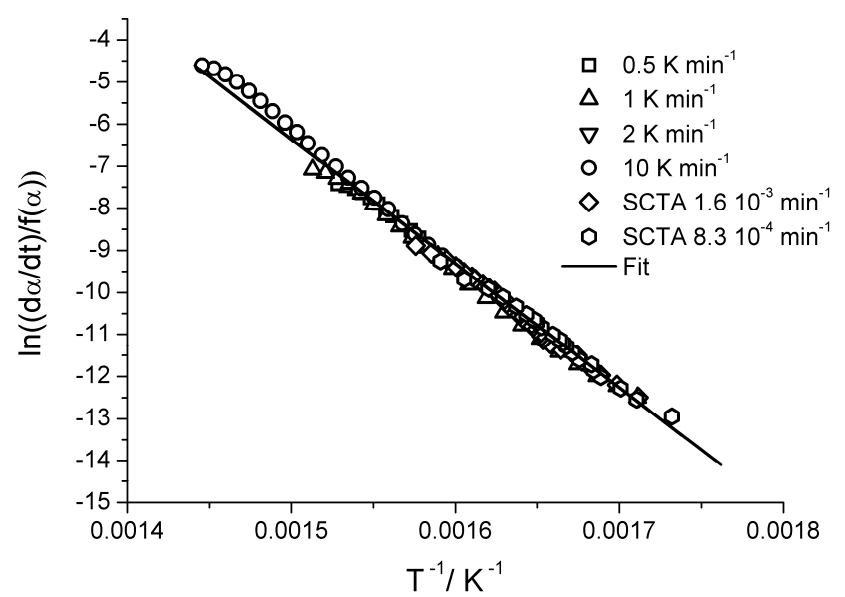

Fig. 8. Combined kinetic analysis of curves included in Fig. 6 by means of Eq. (8) for the $\mathrm{n}$ and $\mathrm{m}$ parameters resulting of the optimization procedure, i.e. $n=0.641$ and $m=-$ 0.646 . 


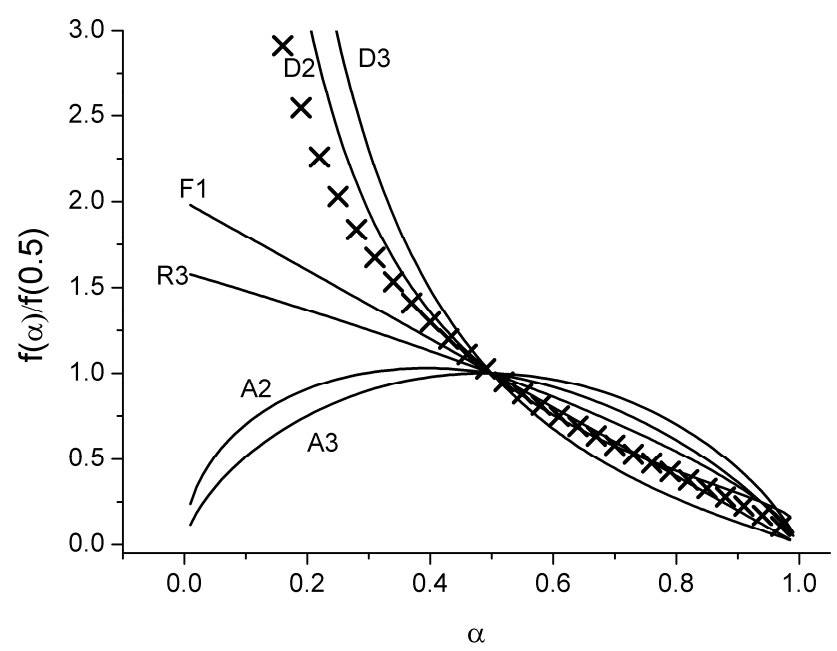

Fig. 9. Comparison of the $\mathrm{f}(\alpha)$ functions (solid lines) normalized at $\alpha=0.5$ corresponding to some of the ideal kinetic models included in Table 1 with the reduced Sestak-Berggren equation (dotted line) and the resulting values of $n$ and $m$ parameters, i.e. $n=0.641$ and $m=-0.646$, for the thermal degradation of polyethylene. 